\title{
Lock-in detection in Raman spectroscopy with charge- shifting CCD for suppression of fast varying backgrounds
}

\author{
Kay Sowoidnich, Michael Towrie, Pavel Matousek
}

\section{Published version information}

This is the peer reviewed version of the following article:

Citation: K Sowoidnich, M Towrie and P Matousek. "Lock-in detection in Raman spectroscopy with charge-shifting CCD for suppression of fast varying backgrounds." J Raman Spectroscopy, vol. 50, no. 7 (2019): 983-995.

\section{DOI: $\underline{10.1002 / \text { irs. } 5597}$}

Which has been published in final form at DOI above. This article may be used for non-commercial purposes in accordance with Wiley-VCH terms and conditions for self-archiving.

Please cite only the published version using the reference above. This is the citation assigned by the publisher at the time of issuing the AAM. Please check the publisher's website for any updates. 


\title{
Lock-in detection in Raman spectroscopy with charge-shifting CCD for suppression of fast varying backgrounds
}

\author{
Kay Sowoidnich, Michael Towrie, Pavel Matousek* \\ Central Laser Facility, Research Complex at Harwell, STFC Rutherford Appleton Laboratory, \\ Harwell Campus OX11 0QX, UK \\ * Corresponding author: E-mail: pavel.matousek@stfc.ac.uk
}

\begin{abstract}
The susceptibility to unwanted light interference significantly limits the potential of Raman spectroscopy by often restricting its applications to environments with controlled ambient light exposure. To overcome this issue, which is particularly relevant to portable, in-field Raman instrumentation, we present an approach based on fast optical lock-in detection using a charge-shifting CCD operation, with charge retention on the chip, for the rejection of varying ambient light interference. In proof-of-concept experiments we used polytetrafluoroethylene (PTFE) as a test sample and Raman spectra were recorded at 830 $\mathrm{nm}$ excitation in the presence of fluorescent or incandescent ambient light. Results demonstrate that the charge-shifting read-out mode (carried out at $1000 \mathrm{~Hz}$ ) has a significant benefit over conventional read-out (limited in practice often to $\lesssim 10 \mathrm{~Hz}$ ) in terms of the removal of dynamically changing ambient light background contributions from Raman spectra. Library-based spectral matching revealed superior reconstruction performance and improved reproducibility for individual spectra obtained using the charge-shifting mode, particularly for interfering lights with spectrally narrow lines and spatially inhomogeneous emission profiles. The approach overcomes fundamental technical limitations of conventional CCD operation and is of particular benefit to mobile Raman systems used in the field in the presence of dynamically changing background light conditions.
\end{abstract}

Keywords: Raman spectroscopy, optical lock-in detection, CCD, ambient light interference, portable instrumentation

\section{Introduction}

Raman spectroscopy is a powerful analytical method for determining the chemical composition of various samples. The technique has a wide range of applications ${ }^{1}$ including material characterization ${ }^{2}$, explosives detection ${ }^{3}$, food analysis ${ }^{4}$, medical diagnosis ${ }^{5}$, forensics ${ }^{6}$, cultural heritage ${ }^{7}$ and extra-terrestrial sample investigation in space missions ${ }^{8}$ to name some. However, due to the weak nature of Raman signals the method is susceptible to ambient light interference, which can easily overwhelm or at least significantly interfere with the Raman signal. Applications of Raman spectroscopy outside 
the usual laboratory environment are therefore often compromised by ambient light (artificial or daylight) which is further complicated by being subjected to additional potential background movement/variations (e.g. due to shadows cast by moving objects, instrument or personnel movements).

In some situations ambient light can be prevented from entering the Raman instrument by physically covering the contact area between sample and probe head, e.g. using a black cloth ${ }^{9}$. This approach has been demonstrated for room lights ${ }^{10,11}$ and sunlight ${ }^{12}$. In the latter case the effect of interfering daylight could also be minimized by either restricting investigations to shaded areas or conducting measurements at times when no sunlight is present, e.g. during evening hours ${ }^{13}$. Using a Raman contact probe it has also been demonstrated that fluorescent ceiling lighting is not a disturbing factor as those contributions do not leak into the system in this measurement geometry ${ }^{14}$. However, the physical blocking of interfering lights is cumbersome and not always feasible, e.g. when extended translucent samples are analyzed or in the case of rapid in-field measurements with handheld instruments or non-contact probes where sensitive or precious specimens are involved.

Alternatively, selected technical solutions have been reported, including the application of short-pulsed lasers in conjunction with gated intensified CCD detectors. The selection of appropriate gate widths, e.g. $10 \mathrm{~ms}$ or shorter, can effectively reduce the ambient light contribution in the recorded Raman spectra ${ }^{15}$. However, high peak powers due to the use of pulsed lasers and added instrumental complexity and costs inherent to this solution hamper its practical deployment, especially in commercial handheld devices. In the special case of ambient daylight interference an excitation in the ultraviolet spectral range below $300 \mathrm{~nm}$ can be beneficial as solar radiation is blocked in this region due to atmospheric ozone absorption ${ }^{16}$. Limitations of this approach are potential sample degradation due to the UV radiation and the fact that any other sources of ambient light apart from sunlight may remain unaffected. The UV region also prevents the use of some special Raman modalities such as Spatially Offset Raman Spectroscopy (SORS) ${ }^{17}$ or transmission Raman spectroscopy ${ }^{18}$ for probing deep inside turbid samples which rely on non-absorbance of the matrix that may not often be achievable in this spectral region. Particularly, the emission of light sources with narrow spectral emission lines compromise considerably the recorded spectra due to appearance of artefacts in Raman spectra ${ }^{19}$.

The issue of ambient lights can also be dealt with by means of special techniques relying on the modulation of the laser excitation wavelength, such as shifted excitation Raman difference spectroscopy (SERDS). In this concept the effective rejection of ambient lights in indoor (incandescent or fluorescent lights) ${ }^{20}$ and outdoor (daylight) ${ }^{21}$ environments is achieved using two alternating, closely spectrally spaced laser emission wavelengths. Furthermore, wavelength-modulated Raman spectroscopy ${ }^{22}$ has been applied for the rejection of fluorescent light interference. Similar to SERDS these approaches rely on the 
fact that the ambient light bands remain practically spectrally static while the Raman spectra are shifted. The modulation is typically performed at relatively low frequencies such as $\lesssim 10 \mathrm{~Hz}$. Subsequent data processing using multivariate statistics can then reject the nonmodulated interference as the Raman signals are identified as, typically, the largest variation in the data set ${ }^{23}$.

Despite the extensive efforts undertaken for the removal of unwanted ambient light contributions, these approaches are most efficient only when mostly static, or slowly modulating backgrounds are present. Several studies using the subtraction of separately acquired background spectra (only containing the interfering light) report issues with artefacts and distortions in the resulting difference spectra due to fluctuations in the background emission ${ }^{24,25}$. The detrimental effect of rapid and irregular background variations can only effectively be addressed with a quasi-simultaneous acquisition of representative background spectra. Due to read-out and analog-to-digital conversion steps required for each recorded spectrum and associated (read-out) noise generated in these steps there is a fundamental technical limitation of conventional CCD based detectors practically limiting the operation speed to less than $\sim 10 \mathrm{~Hz}$.

To address this issue we demonstrate a fast lock-in detection of Raman signals on the CCD with rapid alternating movement of the charge on the CCD chip detecting alternating Raman with background signals and background signal only using a so called CCD charge-shifting modality that has been demonstrated in other areas ${ }^{26,27}$. A similar concept has been demonstrated in Raman spectroscopy by Heming et al. ${ }^{28}$ with a rotating sample cell to differentiate between light-activated and dark-adapted Bacteriorhodopsin specimens. In this case the readout was synchronized and in phase with the rotating cell split into two halves and contained the above species in each half.

In this paper, we demonstrate the lock-in CCD concept for Raman detection in the presence of two representative sources of ambient light. A fluorescent desk light is selected to represent a source with narrow but intense spectral lines while an incandescent halogen light represents a broad and intense light interference such as black body radiation. For both the light sources the influence of static and dynamic illumination conditions has been investigated to evaluate benefits and limitations of this approach.

\section{Materials and Methods}

\subsection{Experimental setup}




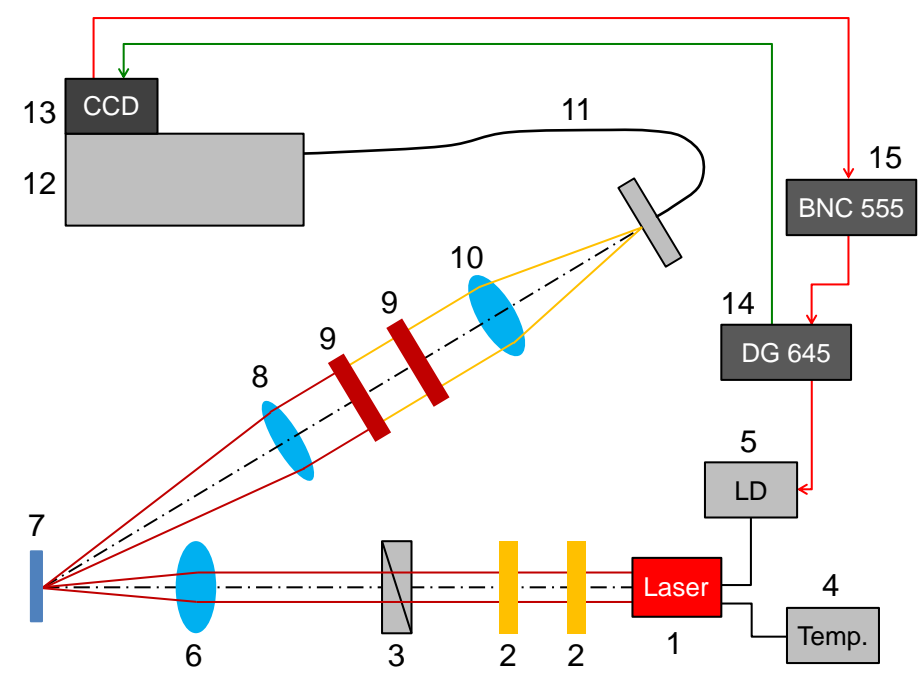

Figure 1: Schematics of experimental setup with $830 \mathrm{~nm}$ microsystem diode laser (1), band pass filter (2), quarter waveplate (3), laser temperature controller (4), laser driver (5), lenses $(6,8,10)$, PTFE sample (7), Raman edge filter (9), optical fiber (11), spectrometer (12), charge-shifting CCD (13), DG645 digital delay generator (14) and BNC model 555 digital delay generator (15), red and green lines with arrows indicate electrical connections for timing synchronization.

A schematic diagram of the experimental setup is presented in Figure 1. A microsystem diode laser (Ferdinand-Braun-Institut, Leibniz-Institut für Höchstfrequenztechnik, Berlin, Germany) emitting a collimated beam at $830 \mathrm{~nm}$ (1) was used as excitation light source. The linearly polarized laser light passes through two bandpass filters (Semrock, Inc.) (2) used to suppress residual amplified spontaneous emission (ASE) and off-central spectral components. The beam is then converted into circularly polarized light by means of a quarter waveplate (WPQ05M-830, Thorlabs, Inc.) (3). Although a circularly polarized light was used in these experiments this had no particular relevance to the detection mode and equally good results would have been obtained with linear or other polarization states. The temperature of the laser module was set to $25^{\circ} \mathrm{C}$ by a temperature controller (5240 TECSource, Arroyo Instruments) (4) and the injection current was controlled by a laser driver (4220-DR LaserSource, Arroyo Instruments) (5). A lens with a focal length of $50 \mathrm{~mm}(6)$ then focused the laser radiation onto a $220 \mu \mathrm{m}$ thick PTFE sample (7). The backscattered radiation was collected by an achromatic lens with a focal length of $100 \mathrm{~mm}$ (8).

Subsequently, two Raman edge filters (Semrock, Inc.) (9) are employed to transmit only the Raman Stokes shifted components while rejecting the elastically scattered laser light. An achromatic lens with a focal length of $60 \mathrm{~mm}$ (10) focused the light into a round-to-linear fiber bundle (BFL200LS02, Thorlabs, Inc.) (11) which then transferred the detected light into the spectrometer (Holospec 1.8i, Kaiser Optical Systems, Inc.) (12) with attached custom made charge-shifting CCD (DU420A-BR-DD-9UW, Andor Technology, S/N CCD-19689) (13) thermo-electrically cooled down to $-70^{\circ} \mathrm{C}$ and controlled by a PC running Andor Solis software (version 4.28.30052.0, Andor Technology). The CCD had no internal nor external shutter. The back and forth charge shifting mode of operation was facilitated by the Solis software which had a feature built in to our custom specifications enabling the overall number of cycles and the number of shifted rows to be specified. Both the laser and Raman 
collection arms were shielded from ambient light using a black aluminum foil (BKF12, Thorlabs, Inc.).

To synchronize the laser emission with the charge-shifting CCD read-out one of the output ports of a digital delay generator (DG645, Stanford Research Systems) (14) was connected to the external trigger input of the CCD (13) while another port was connected to the modulation input of the laser driver (5). The shutter output of the CCD was connected to the input of a second digital delay generator (Model 555, Berkeley Nucleonics Corporation) (15). The output of the Model 555 delay generator was connected to the "Inhibit" input of the DG645 delay generator to enable laser emission only during the charge-shifting procedure but not during the final read-out phase.

\subsection{Charge-shifting method}

The key feature of the charge-shifting approach is that the usual read-out and digitization steps, which slow down the acquisition and introduce additional (read-out) noise to the data, are eliminated during the cyclic acquisition and only performed once, e.g. after many thousand cycles, at the end of the entire measurement. In the charge-shifting CCD read-out mode an external trigger signal (frequency of $1000 \mathrm{~Hz}$ in this case) starts the first cycle in which a defined area on the CCD chip (called area 1 hereafter) is illuminated, e.g. by Raman scattered light from the sample and ambient light contributions (Figure 2, step a1). When receiving the following trigger signal the second cycle starts with the charge accumulated in area 1 being electronically shifted to a non-illuminated CCD area (i.e. 80 rows up) and subsequent illumination of the CCD, e.g. by ambient light only (Figure 2, steps a2 and a3). It is important to note that physically the same pixels on the CCD chip are illuminated during this second cycle but that the charge is accumulated in a different area of the electronic register (called area 2 hereafter). The next trigger signal starting cycle three shifts both areas downwards by the same number of rows (Figure 2, step a4). This causes area 2 to be moved to a non-illuminated range of the CCD while area 1 will be illuminated again, e.g. by Raman scattered light from the sample and ambient light contributions (Figure 2, step a5). The subsequent trigger signal will then shift both areas upwards by 80 rows (Figure 2, step a6) moving area 2 back to the illuminated region of the CCD. The sequence of alternate illumination of the CCD in either area 1 or area 2 is repeated 5000 times, corresponding to a total acquisition time of 5 seconds. Finally, the accumulated charge on the CCD chip (operated in image mode with a vertical binning of 16 pixels applied) is read out and digitized. The charge-shifting process is usually repeated several thousand times until sufficient charge is built up for it to be read or until CCD saturation level is approached. Due to the absence of any digitization steps within the cycles and the involvement of only charge movement up and down on the CCD chip the shifting process can be facilitated at frequencies in the kilohertz range with a high performance spectroscopic CCD with 
practically no noise penalty on shifted charge, which can be kept accumulating in each CCD area throughout the entire measurement. In this way, the charges accumulated in both the individual areas on the CCD after several thousand of cycles will permit the retrieval of spectra with uncompromised signal-to-noise ratio. The final readout will only introduce read out noise once to the already high intensity and good quality spectra which given the charge signal height at that stage can be typically negligible. For charge-shifting as well as for the conventional operation mode, in Andor Solis software a vertical pixel shift speed of $8.25 \mu \mathrm{s}$ (the fastest possible option with the CCD camera used), a horizontal pixel shift readout rate of $100 \mathrm{kHz}$ at 16-bit (the fastest possible option with the CCD camera used) and a preamplifier gain of $1.7 x$ were used.

As the illumination condition for area 1 can be either with the laser on or with the laser off there exist two different illumination geometries (see Figure 2), called "a" (laser emission in area $1 /$ top area in final image) and " $b$ " (laser emission in area 2 / bottom area in final image). Spectra were recorded in pairs of "a" and " $b$ " geometries resulting in a total acquisition time of 10.18 seconds (10,000 shifting cycles of 1 ms duration each plus twice 88.24 ms CCD read out and digitization time).
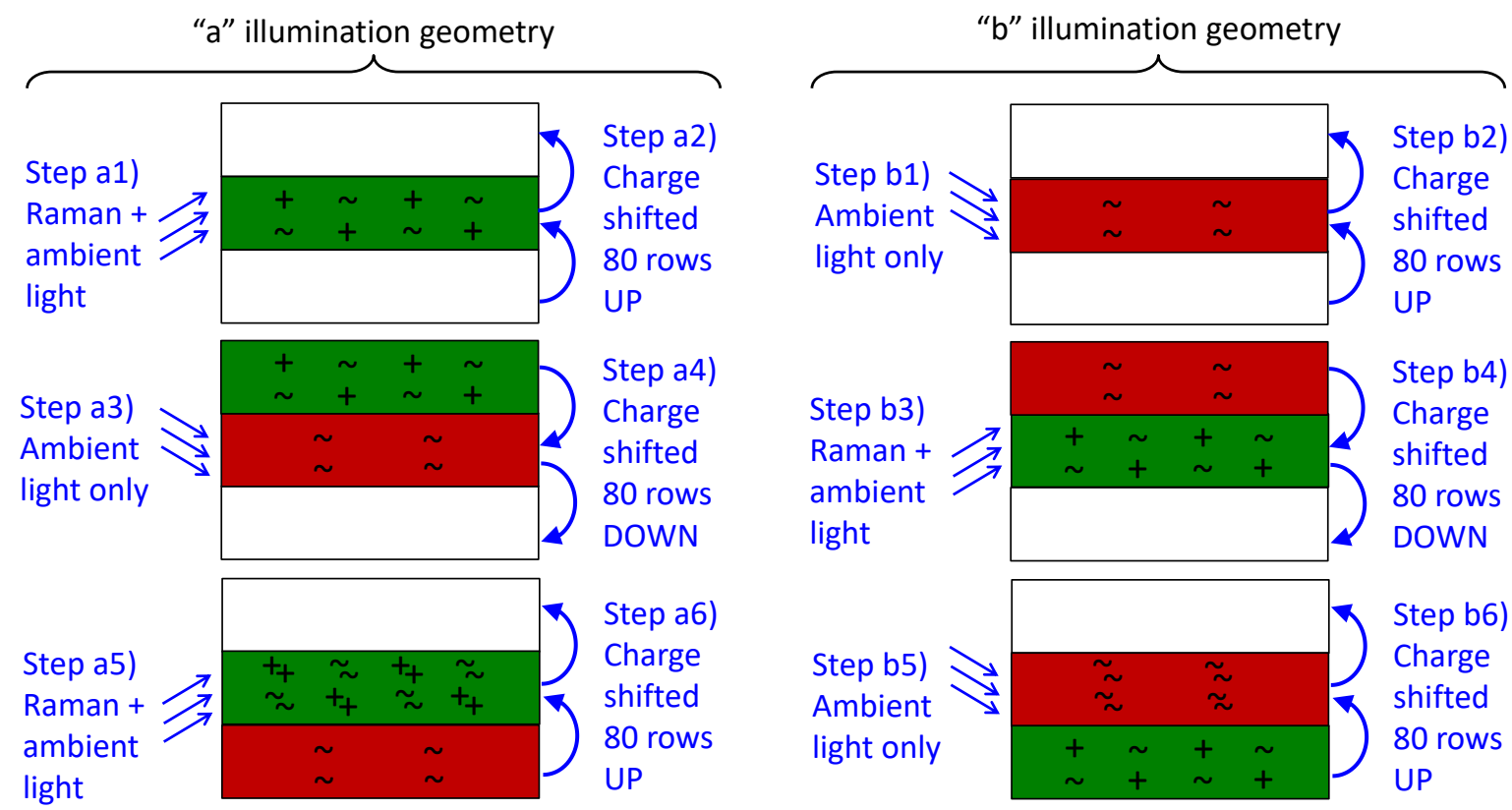

Figure 2: Scheme of the charge-shifting process illustrated for the initial cycles in both available illumination geometries. Initial steps for "a" illumination geometry (left image sequence): Accumulation of charges in active area caused by Raman and ambient light illumination (step a1) followed by shifting of these charges to a non-illuminated (top) storage area (step a2), Accumulation of charges due to ambient light illumination in active area (step a3) and subsequent shift of these charges down to a second (bottom) non-illuminated storage area (step a4), Repetition of cycle one by accumulating more charges caused by Raman and ambient light illumination in active area (step a5) followed by shifting of these charges to the top storage area (step a6). The corresponding initial steps for the "b" illumination geometry are depicted in the right image sequence. Symbols denote charges on CCD chip created by illumination with Raman (+) and ambient light ( ). 
The laser driver is set to an injection current of $560 \mathrm{~mA}$, which is below the lasing threshold (corresponding to "laser off" state), and modulated by the DG 645 at a frequency of $500 \mathrm{~Hz}$, i.e. firing only when the charge on the CCD is in an identical position (top or bottom area) as it was for the previous laser pulse. Applying a modulation voltage of $1 \mathrm{~V}$ leads to a laser injection current in the "laser on" state of $660 \mathrm{~mA}$ resulting in an optical power at the sample position of $52 \mathrm{~mW}$. To avoid laser emission during the time required to shift the charge 80 rows up and down (which would lead to signal mixing in the two areas), a pulse delay between CCD trigger signal and laser pulse output of $680 \mu \mathrm{s}$ was applied. Limited by the time available until the subsequent pulse arrives (i.e. $1 \mathrm{~ms}$ ), the laser pulse width was set to $319 \mu \mathrm{s}$.

The external trigger signal required by the charge-shifting CCD was provided by the DG645 delay generator. It should be noted that the corresponding pulse output is not affected by the "Inhibit" function thus delivering a continuous pulse train to the "EXT TRIG" input of the $\mathrm{CCD}$ at the selected frequency of $1000 \mathrm{~Hz}$. When the charge-shifting sequence is started in the Solis software, the CCD "Shutter" output will send a trigger signal to the input of the Model 555 delay generator. This delay generator is set to a TTL high configuration activating the "Inhibit" function for the laser output of the DG645 delay generator when no trigger signal is received, i.e. the laser is in the "off" state. Upon receipt of the trigger signal from the CCD the Model 555 delay generator outputs a pulse of selected time (i.e. 5 seconds) to disable the "Inhibit" function of the DG645 and consequently enable the laser output for that particular duration. The entire sequence is completed when a pre-set number of cycles is reached (selected in Solis software). The CCD is then read by binning the charge vertically into a total number of 16 segments containing 16 pixels each. From these segments two separate spectra, one containing Raman signal with background (e.g. segments 1-4 corresponding to CCD pixels 1-64) and the other with background signal only (e.g. segments 6-9 corresponding to CCD pixels 81-144) are calculated by averaging.

\subsection{Conventional read-out}

Spectra were also recorded in conventional CCD read-out for comparison using subacquisition times of $50 \mathrm{~ms}, 100 \mathrm{~ms}$ and $500 \mathrm{~ms}$. Two adjustment steps were performed to match the illumination conditions in both charge-shifting and conventional read-out mode. First, the total amount of ambient light striking the CCD during total acquisition time was matched by selecting a suitable number of acquisitions in the conventional read-out mode. Second, for the given number of total acquisitions the total number of laser photons striking the sample during individual acquisitions was matched by selecting an appropriate laser pulse width. This resulted in the laser illumination times being somewhat shorter than otherwise achievable in the conventional sub-acquisitions $(26 \mathrm{~ms}$ instead of $50 \mathrm{~ms}, 43 \mathrm{~ms}$ instead of $100 \mathrm{~ms}$ and $177 \mathrm{~ms}$ instead of $500 \mathrm{~ms}$ for the $50 \mathrm{~ms}, 100 \mathrm{~ms}$ and $500 \mathrm{~ms}$ read- 
outs, respectively) making the overall conventional read-outs correspondingly longer than would otherwise be achievable (see Table 1). To avoid illumination of the CCD during the read-out phases, the position of the laser pulse was adjusted to be at the center of the individual acquisition time window, i.e. an appropriate laser pulse delay relative to the CCD trigger pulse was chosen. Table 1 presents an overview of the relevant acquisition parameters. To mimic the read-out conditions of the charge-shifting mode, spectra were acquired in multi-track mode using 4 tracks containing 16 vertical pixels each. Furthermore, the CCD was externally triggered by the DG645 delay generator to record a kinetic series with adjacent spectra corresponding to "laser on" and "laser off" state.

\subsection{Ambient light sources}

As a broadband light source a $35 \mathrm{~W}$ halogen light bulb was used directly illuminating the sample area. Due to its lower intensity the light emitted from a compact fluorescent light tube (having several narrow emission lines) was reflected towards the sample surface using a mirror in addition to the direct illumination of the sample area. The intensity of both ambient lights was set so that the ratio of the main PTFE Raman band at $734 \mathrm{~cm}^{-1}$ to the most intense ambient light signal was in the range of 0.008 to 0.026 (see Figure 3 ). In the static illumination condition the ambient light interference was recorded without any modulation whilst under dynamic illumination the light intensity was modulated by waving the hand of the operator immediately beneath the corresponding light source crudely mimicking the movement of personal, other objects around the measurement area or the light source itself. For both light sources in conventional and charge-shifting read-out under static and dynamic illumination 6 repeat sets of experiments were performed with 10 individual spectra recorded each.

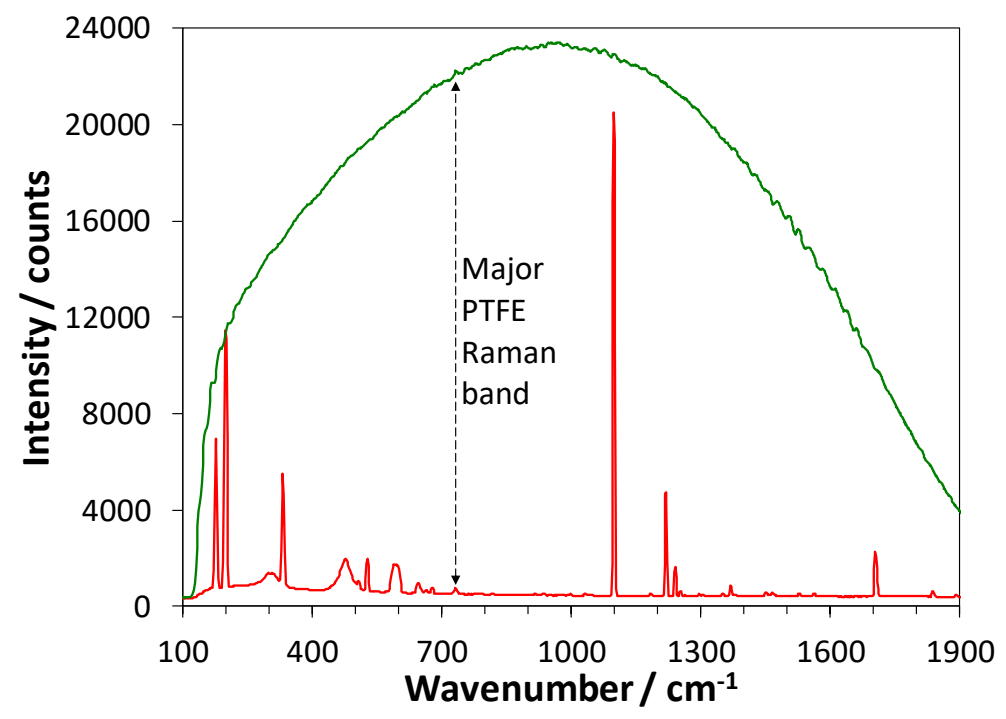


Figure 3: Raman spectra of PTFE recorded with additional fluorescent (red curve) and incandescent (green curve) light interference under static illumination conditions exemplifying the severity of typical background levels used relative to Raman signal. Displayed are averages of 10 individual spectra recorded in charge-shifting mode.

\subsection{Data analysis}

For the conventional read-out the intensities acquired on the selected illumination track (vertical pixels 81-144) were averaged and individual spectra for both cases ("laser on" / "laser off") in the kinetic series were accumulated leading to average spectra, i.e. one containing the PTFE Raman signal and the ambient light signal and the other containing only the ambient light contribution. The minimum values from each of the two spectra were then subtracted, following by normalization to the most intense feature of the ambient light source, i.e. the top of the sharp signal at $1098 \mathrm{~cm}^{-1}$ for the fluorescent light (mean of 3 data points) or the top of the broad emission profile between $947 \mathrm{~cm}^{-1}$ and $973 \mathrm{~cm}^{-1}$ for the incandescent light (mean of 15 data points). Finally, the difference between two corresponding spectra was taken.

The charge shifting spectra were processed in a similar way as the spectra in conventional read-out. However, here intensities acquired in the top (vertical pixels 81-144) and bottom area (vertical pixels 1-64) were averaged in the initial step and after difference computation (top area minus bottom area) a final reconstruction step was added by subtracting the " $b$ " spectrum from the "a" spectrum.

To obtain a reference spectrum of the PTFE tape all ambient lights were turned off and two high signal-to-noise-ratio spectra were recorded using an integration time of 10 seconds and 10 individual spectra were averaged each. Subsequently, the spectrum corresponding to the "laser off" state (560 mA injection current) was directly subtracted from the one corresponding to the "laser on" state (660 mA injection current).

From the spectra with contributions from both the PTFE Raman signal and the ambient light the intensities of characteristic spectral features were determined. The PTFE Raman band intensity of the $C-F$ and $C-C$ symmetric stretch band 29 at $734 \mathrm{~cm}^{-1}$ was calculated as the average of the intensity of three points at the peak center above the baseline as determined by the average intensities of 5 points left and right to the band using Microsoft Excel (Microsoft, Redmond, WA). The ambient light intensity for the fluorescent light was determined in an identical way but using the most intense emission line at $1098 \mathrm{~cm}^{-1}$. In the case of the incandescent light the maximum intensity of the broad emission was calculated by subtracting the average intensity of the background in the range between $70 \mathrm{~cm}^{-1}$ and 98 $\mathrm{cm}^{-1}$ from the mean intensity in the region between $947 \mathrm{~cm}^{-1}$ and $973 \mathrm{~cm}^{-1}$. To calculate the PTFE signal-to-noise-ratio ( $\mathrm{S} / \mathrm{N}$ ratio) of the recovered spectra the background noise value was determined in the spectral range from 810 to $860 \mathrm{~cm}^{-1}$ that is free from signals either from PTFE or any of the fluorescent light emission lines. 
For quantitative assessment of spectral reconstructions the hit quality index (HQI) 30 was used as indicator of spectral similarity between individual reconstructed spectra and the PTFE reference spectrum. The HQI is indicating the quality of reconstructed spectra and high $\mathrm{HQI}$ values are particularly relevant to spectral identification. To account for potential variations in the ambient light intensity level, which are inevitable particularly under dynamic illumination conditions, the HQI is plotted against the PTFE signal-to-ambient-lightintensity ratios.

\section{Results and discussion}

\subsection{Fluorescent light interference}

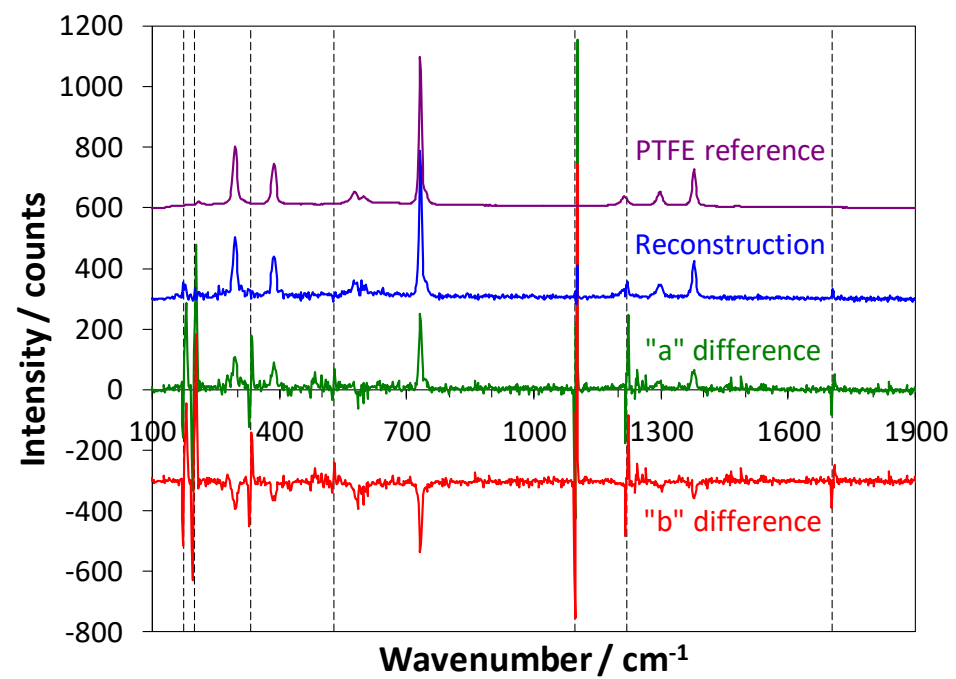

Figure 4: Recorded charge-shifting spectra under static fluorescent light illumination. To calculate the "Reconstruction" spectrum the " $b$ " difference spectrum is subtracted from the " $a$ " difference spectrum. The PTFE reference spectrum is displayed for comparison. Vertical dashed lines indicate spectral positions of fluorescent light emission lines and spectra are vertically offset for clarity.

In case of the conventional read-out the spectral reconstruction can be performed simply by scaled subtraction of the "laser off" spectra from the "laser on" spectra. For the chargeshifting read-out, however, the simplest scaled subtraction of the two separate tracks recorded, i.e. Raman plus ambient light and ambient light only, leaves pronounced residuals of the fluorescent light emission lines (green curve in Figure 4). The spectral pattern of the residuals resembles the form of the first derivative, indicating that a shift of the ambient light emission lines may have been present during the charge-shifting process. This effect can be understood in terms of the well-known "smile" pattern inherent to imaging spectrographs where the plane grating results in a curved image of the straight entrance slit 31.

In the conventional read-out this does not pose an issue as the charge is only shifted in one direction and only for short duration of time, i.e. during the actual read-out phase (duration 
ca. $90 \mathrm{~ms}$ per exposure). Therefore, consecutive spectra recorded with and without the laser being turned on experience exactly the same amount of distortion and the individual contributions cancel out during subtraction. In the charge-shifting mode, however, the charge is shifted in both directions during the exposure phase, the shift is multiply repeated and a relatively large number of 80 rows are shifted and consequently the distortions caused by the "smile" effect cannot fully be compensated. It should be noted that the effect is small in relative terms as the maximum residual intensity around $1100 \mathrm{~cm}^{-1}$ (green curve in Figure 4) amounts to less than $6 \%$ of the original intensity of the fluorescent light emission line in the top and bottom track spectra.

Nevertheless, this effect can be eliminated by the following simple methodology. Determined by the starting trigger signal, the Raman scattered light originating from the PTFE sample is either imaged on the top track ("a" spectrum) or on the bottom track ("b" spectrum) in charge-shifting mode. Subtracting the bottom from the top track in both cases results in a positive Raman intensity for the "a" differences while for the "b" difference (red curve in Figure 4) a negative Raman intensity is obtained. As the spectral distortions are equal in both cases they can effectively be eliminated by subtracting both difference spectra from each other leading to the reconstructed PTFE spectrum (blue curve in Figure 4) with Raman intensities added to each other. A comparison of the reconstructed spectrum with the reference spectrum (purple curve in Figure 4) highlights the good quality of the obtained spectrum with only very minor residual distortions being left.

\subsubsection{Recovered PTFE spectra}

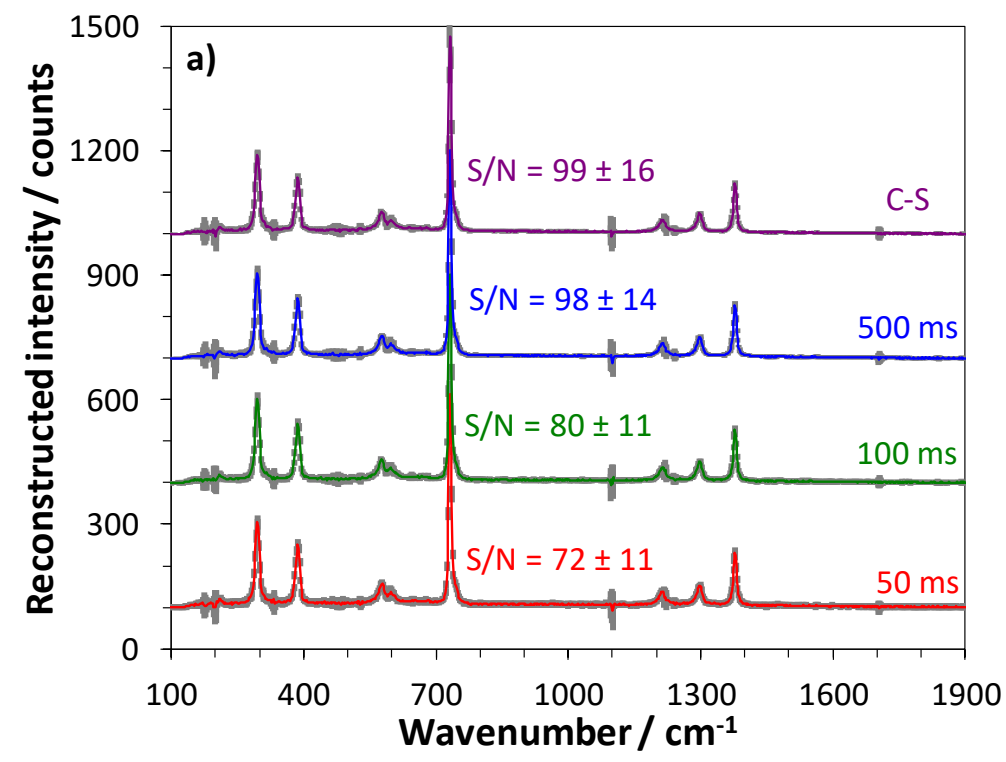




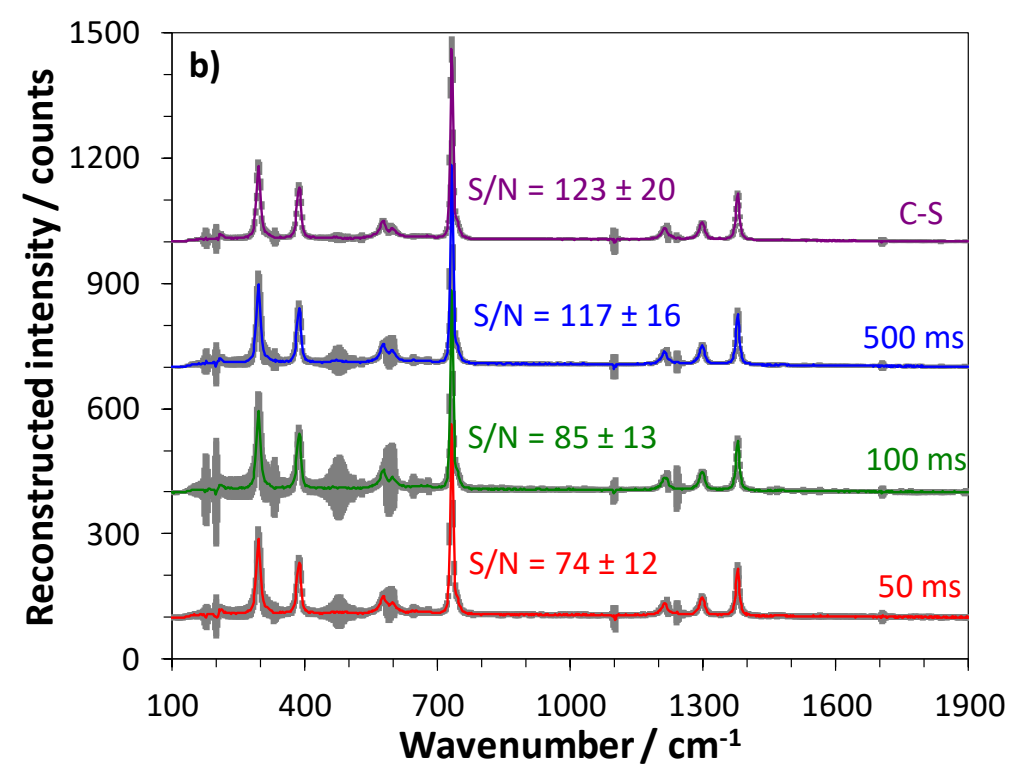

Figure 5: Average and standard deviation (gray areas) of 60 recovered spectra of PTFE layer for fluorescent light interference under static (a) and dynamic (b) illumination conditions in conventional and charge-shifting (C-S) read-out modes, spectra are vertically offset for clarity, $\mathrm{S} / \mathrm{N}$ ratios are given as mean \pm standard deviation of 60 spectra each.

The average reconstructed spectra under static illumination conditions show very similar patterns in both conventional and charge-shifting (C-S) read-out as expected (see Figure 5a). The fluorescent light interference has effectively been removed in all cases with only minor contributions from the formerly very intense emission lines remaining with a good reproducibility of reconstructed spectra being achieved (as indicated by the standard deviation). Regarding the $\mathrm{S} / \mathrm{N}$ ratios of the reconstructed spectra, however, there are distinct differences between individual acquisition times in conventional read-out and the charge-shifting mode. This effect can be explained in terms of read-out noise dependent on the number of individual acquisitions used. While in charge-shifting mode only two readouts took place, the number of read-out cycles is 18,74 and 122 for sub-acquisition times of 500, 100 and $50 \mathrm{~ms}$ in conventional mode, respectively. At $500 \mathrm{~ms}$ acquisition time, due to the relatively low number of acquisitions, the detrimental effect of accumulated read-outnoise vanishes as obtained $\mathrm{S} / \mathrm{N}$ ratios are identical to the charge-shifting mode.

As shown in Figure 5b, under dynamic illumination conditions the standard deviation of 60 reconstructed spectra (indicated by the gray areas in each case) is much larger for the conventional read-out mode compared to the charge-shifting read-out. Particularly in the case of a sub-acquisition time of 100 ms the residuals of fluorescent light emission lines are prominent. Further tests have shown that this observation is due to the relatively large active surface of the fluorescent light bulb (2 separate U-shaped discharge tubes with 10 $\mathrm{mm}$ diameter and $94 \mathrm{~mm}$ length each) resulting in a spatial variation of the spectral emission profile across its length. When moving the hand between light source and experimental setup under dynamic illumination conditions the collection optics captures slightly different spectral distribution at different times. As the hand movement is not 
synchronized with the CCD read-out frequency the spectral distortions are not evenly distributed onto adjacent spectra in the kinetic series. Consequently, the spectral reconstruction process is not capable of removing these distortions effectively. At the applied 'hand waving' frequency sub-acquisition times of $50 \mathrm{~ms}$ and $500 \mathrm{~ms}$ resulted in better reconstruction performance but it should be noted that this may not be the case for different modulation frequencies. The only way to overcome this fundamental issue is the ability of the charge-shifting mode operated at a frequency of $1000 \mathrm{~Hz}$ to effectively distribute fast variations in the background level evenly onto both active areas on the CCD chip so that subsequent spectral reconstruction can efficiently remove these disturbances. Due to the lower absolute intensity of fluorescent light striking the CCD the $\mathrm{S} / \mathrm{N}$ ratios increased in all cases compared to the static case but still show the same trend already observed for the static illumination conditions.

\subsubsection{Quantitative assessment of spectral reconstruction}

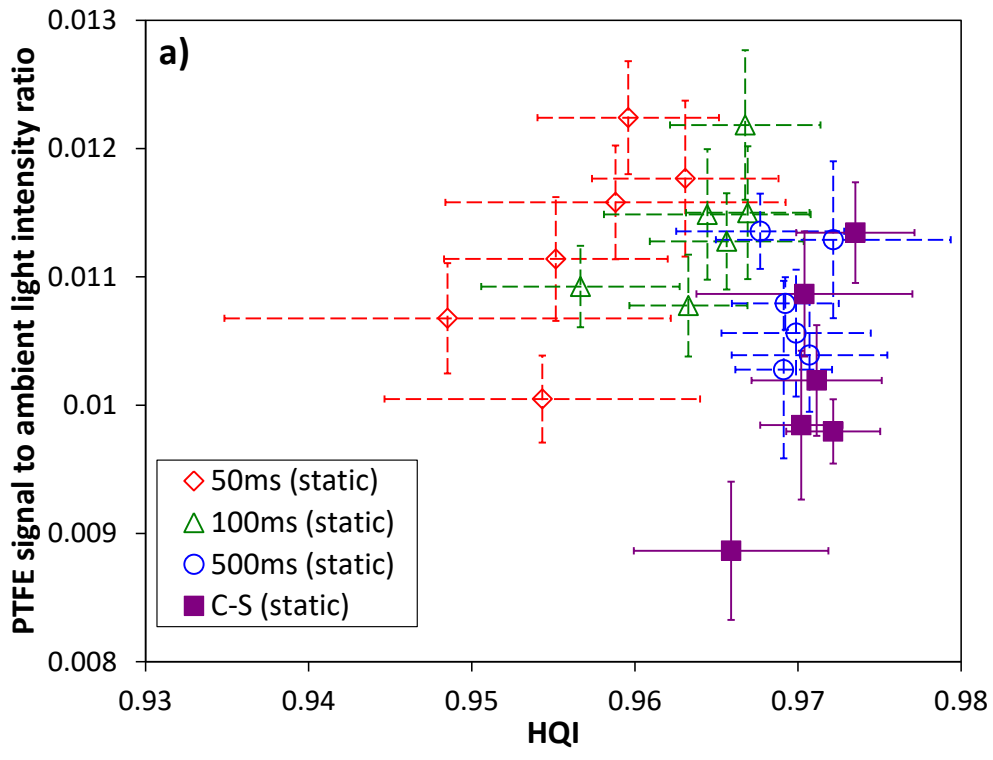




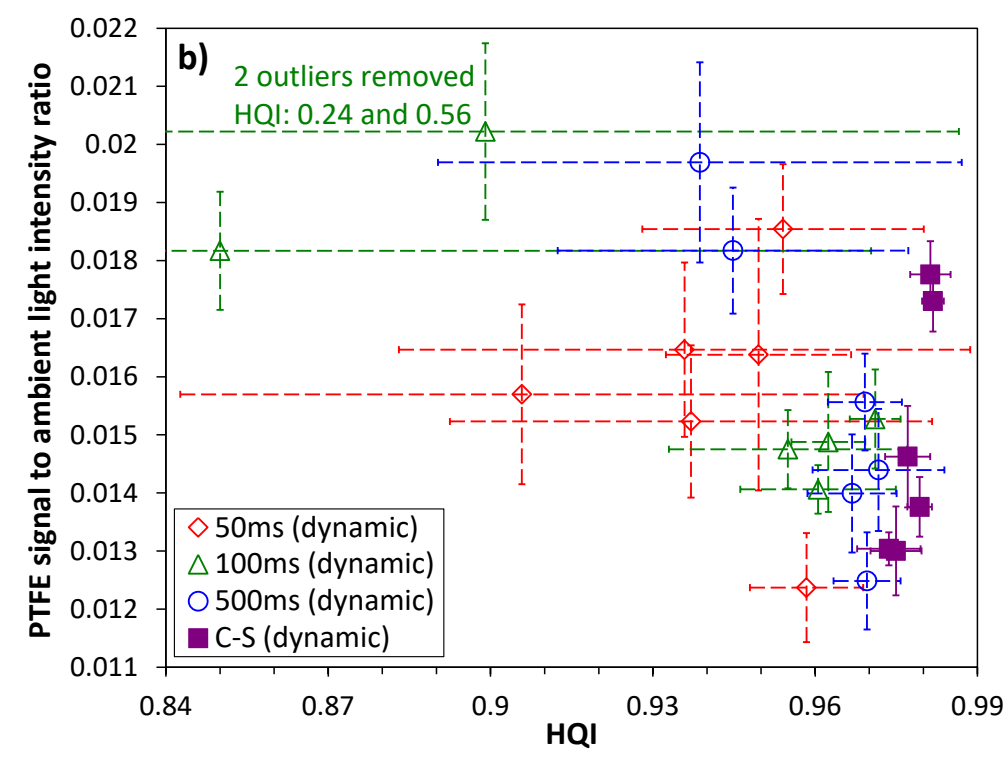

Figure 6: PTFE Raman signal intensity to ambient light intensity ratio plotted against HQI for fluorescent light interference for conventional and charge shifting (C-S) modes under static (a) and dynamic (b) illumination conditions; error bars represent standard deviation of ten individual spectra unless stated otherwise.

For a quantitative assessment of the recovered spectra in conventional and charge-shifting read-out the PTFE to fluorescent light intensity ratio has been plotted against the hit quality index (HQI) with a perfect spectral match represented by a score of 1.

Under static illumination conditions there is not much difference in reconstruction performance between the conventional read-out and the charge-shifting mode with very good reconstructions being achieved in all cases (see Figure 6a). For the conventional readout, however, there is a trend for better reconstructions with increased sub-acquisition time. The spectra reconstructed from the series of 18 individual spectra recorded at $500 \mathrm{~ms}$ show the best performance and are, at comparable PTFE signal to ambient light intensity ratios, virtually identical to the spectra reconstructed from charge-shifting acquisition. As there is no significant amount of shot-noise added to the spectra by means of the fluorescent light emission with its almost flat baseline this effect can be understood in terms of increased amounts of accumulated read-out noise for shorter sub-acquisition times. As expected for non-varying backgrounds, the conventional read-out mode at frequencies in the range of $1.7-7.5 \mathrm{~Hz}$ is capable of rejecting those static contributions effectively.

Figure $6 \mathrm{~b}$ shows that in the case of dynamic illumination, however, the charge-shifting method is superior in addressing rapidly varying modulations of ambient light interference resulting in the best reconstruction (highest HQI) and the best reproducibility (lowest standard deviation within each set of 10 spectra). It should be noted that the reconstruction of two spectra recorded at $100 \mathrm{~ms}$ exposure time under conventional conditions led to very poor outcomes and these two outliers have been removed for clarity of presentation. The results demonstrate that conventional read-out that is limited to frequencies of less than 10 
$\mathrm{Hz}$ is not able to efficiently and reproducibly address irregular modulations/variations of ambient light interference.

In contrast to the static illumination conditions there is an apparent negative correlation between the PTFE signal to fluorescent light background ratio and the HQI for the conventional read-out at all investigated sub-acquisition times. For the charge-shifting readout, however, the opposite effect, i.e. better reconstruction performance with increasing PTFE to ambient light intensity ratio, can be observed. It is believed that this observation is due to the different abilities of the read-out modalities to deal with varying backgrounds. For the charge-shifting mode, which can effectively reject rapidly varying ambient light contributions, a lower but fluctuating fluorescent light intensity will result in a better reconstruction. Due to the fast modulation at $1000 \mathrm{~Hz}$ the residual distortions in the reconstructed spectra are reduced as fluorescent light is evenly distributed (sampled) between the two different areas of the electronic CCD register used to acquire the "laser on" and "laser off" spectra. For the conventional read-out the higher degree of modulation causing a lower absolute but more fluctuating fluorescent light intensity (due to less averaged background being accumulated) will result in more pronounced residual distortions in the reconstructed spectra as this read-out modality has limited capability to deal with rapid variations (see also Figure 5b). Effectively, due to the irregular nature of the applied modulation the fluorescent light contributions are unevenly distributed between adjacent spectra corresponding to "laser on" and "laser off" state in the recorded kinetic series which will inevitably cause distortions in the reconstructed spectra. Due to the narrow-band spectral features of the fluorescent light these pronounced deviations from the PTFE reference spectrum will have a detrimental effect on the reconstruction performance ultimately resulting in lower HQI values.

In terms of material identification a typical minimal HQI used for confirmation purposes is 0.9530 . Applying this threshold value the correct classification rates for the PTFE sample under investigation were $57 \%, 68 \%$ and $82 \%$ for the conventional read-out with subacquisition times of 50, 100 and $500 \mathrm{~ms}$, respectively. In contrast, the superior spectral reconstruction performance of the charge-shifting technique led to $100 \%$ correct classification rate, i.e. the sample was identified as PTFE in 60 out of 60 cases. For the identification of mixtures or quantification purposes higher quality spectra are always more desirable as they lead to more accurate results and as such the charge shifting-mode would be expected to be the most effective.

One major difference between the two read-out modes tested is that the reconstruction performance decreases from static to dynamic illumination conditions for all investigated sub-acquisition times in conventional read-out (reductions of 1.0-3.2 \%) while the opposite is the case for the charge-shifting mode (increase by $0.8 \%$, see Figure 7 ). Furthermore, the reproducibility of spectral reconstructions is reduced in the conventional mode by factors of $4.3,12.2$ and 5.4 for sub-acquisition times of $50 \mathrm{~ms}, 100 \mathrm{~ms}$ and $500 \mathrm{~ms}$, respectively while 
no change is observed for the charge-shifting read-out. This result can be explained by two counteracting effects. In the static illumination geometry the accumulated fluorescent light intensity during the measurement is higher (but stable) whereas under dynamic illumination conditions a varying (but overall lower) amount of ambient light is captured. As the chargeshifting mode (operated at $1000 \mathrm{~Hz}$ ) has a superior ability to deal with modulated backgrounds, the Raman spectra acquired with smaller absolute fluorescent light intensity is rendered with a better spectral reconstruction under dynamic illumination conditions. In contrast, the conventional read-out is not able to handle background variations that well and so in this case the benefit of lower absolute intensities cannot be exploited fully leading to decreased reconstruction performance and increased variation.

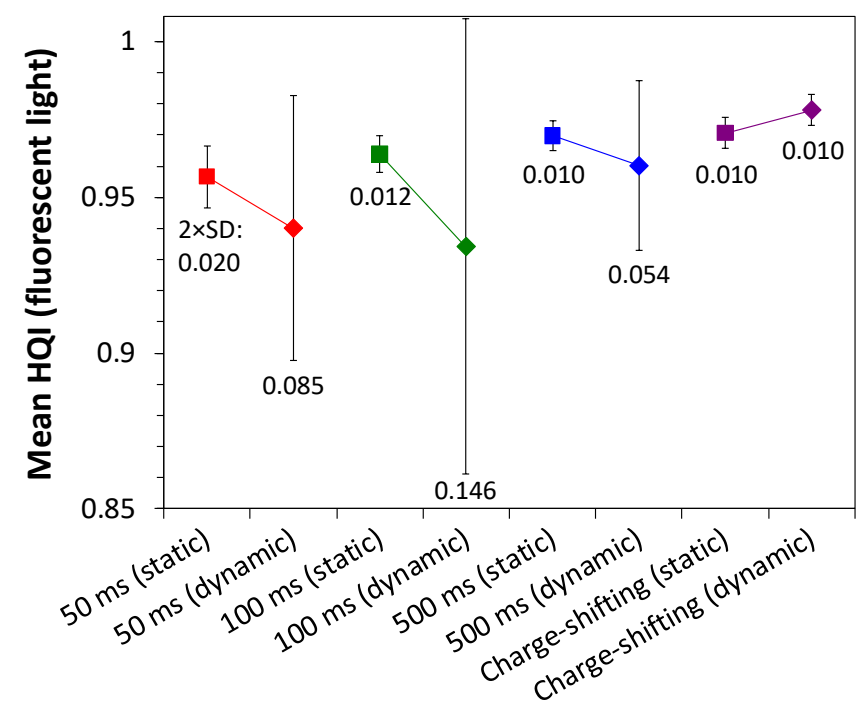

Figure 7: Overall comparison of mean HQI and standard deviation for 60 spectra recorded under fluorescent light illumination (static and dynamic conditions) in conventional and charge-shifting read-out modes. The numbers given are twice the standard deviation and represent the total length of the error bars.

\subsection{Incandescent light interference}


3.2.1. Recovered PTFE spectra
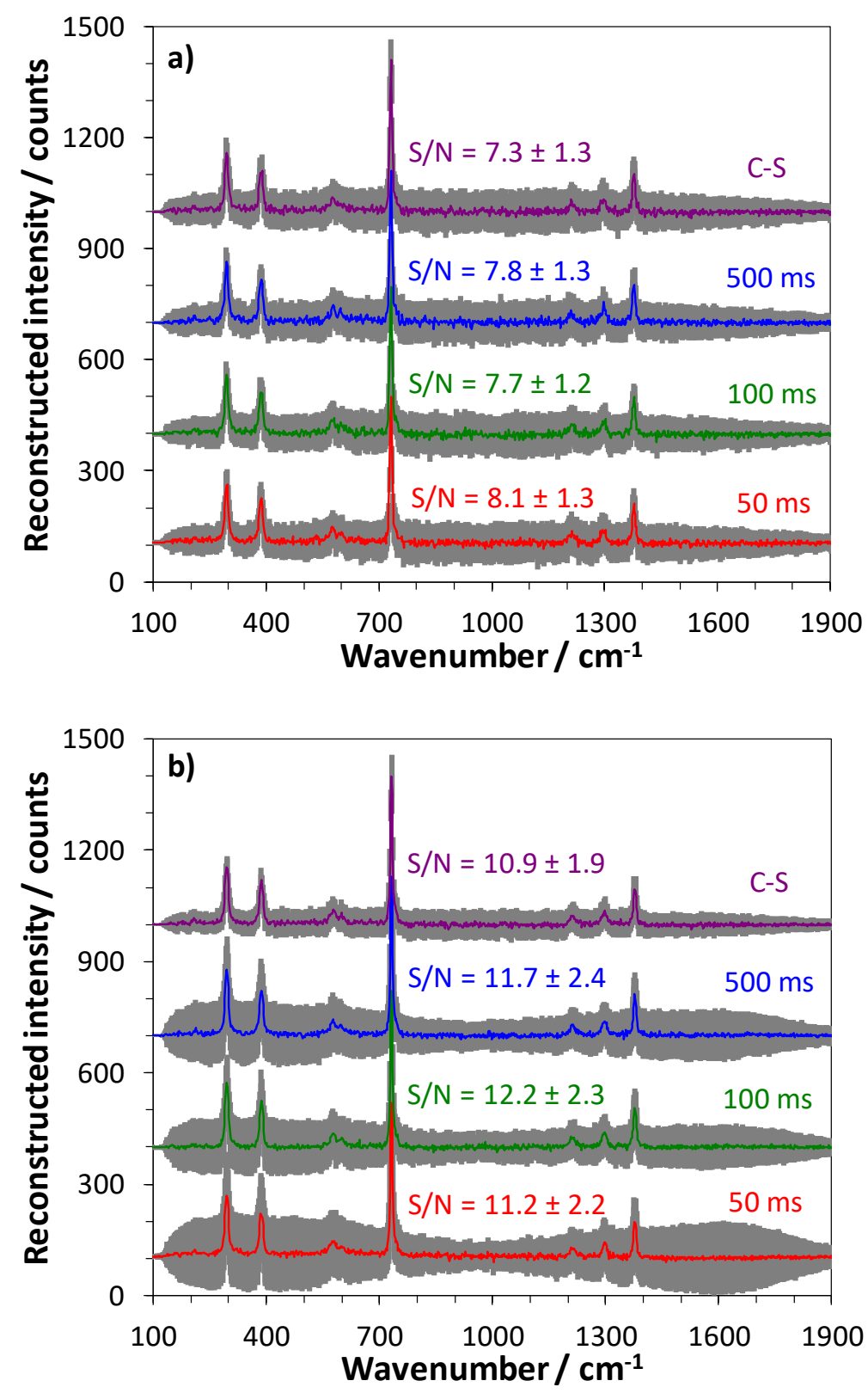

Figure 8: Average and standard deviation (gray areas) of 60 recovered spectra of PTFE layer for incandescent light interference under static (a) and dynamic (b) illumination conditions in conventional and charge-shifting read-out modes, spectra are vertically offset for clarity, $\mathrm{S} / \mathrm{N}$ ratios are given as mean \pm standard deviation of 60 spectra each.

The incandescent light source has a broad and intense spectral emission adding a significant background to the recorded spectra at all wavenumbers (see Figure 3). The reconstructed spectra under static illumination conditions (presented in Figure 8a) show S/N ratios about one order of magnitude lower compared to the fluorescent light of comparable peak intensity (see Figure 5a). In contrast to the fluorescent light in this case there is no trend observable for the $\mathrm{S} / \mathrm{N}$ ratios with respect to the sub-acquisition times in conventional readout mode. Owing to the spectral properties of the incandescent light the amount of photon 
shot noise contained in the spectra is much larger than the accumulated read-out noise and can therefore be considered as dominant source of noise.

Under dynamic illumination conditions spectral distortions introduced by the modulation of the light intensity contribute as a further source of variation. In the conventional read-out mode this effect causes the standard deviation (gray areas in Figure 8b) to increase in the low and high wavenumber regions (i.e. outside the region where normalization is performed for the spectral reconstruction) relative to static illumination due to the limited ability of this configuration to deal with varying backgrounds. For the charge-shifting mode, however, the ability to effectively reject that type of variation leads to a significantly reduced standard deviation as the overall light intensity is reduced compared to the static illumination conditions. Regarding the $\mathrm{S} / \mathrm{N}$ ratios governed by fine pixel-to-pixel noise there is no difference observable between the various read-out configurations. The absolute values, however, are increased by factors of about 1.4-1.6 compared to the static illumination conditions as the overall incandescent light intensity is reduced resulting in a lower shot noise contribution.

\subsubsection{Quantitative assessment of spectral reconstruction}

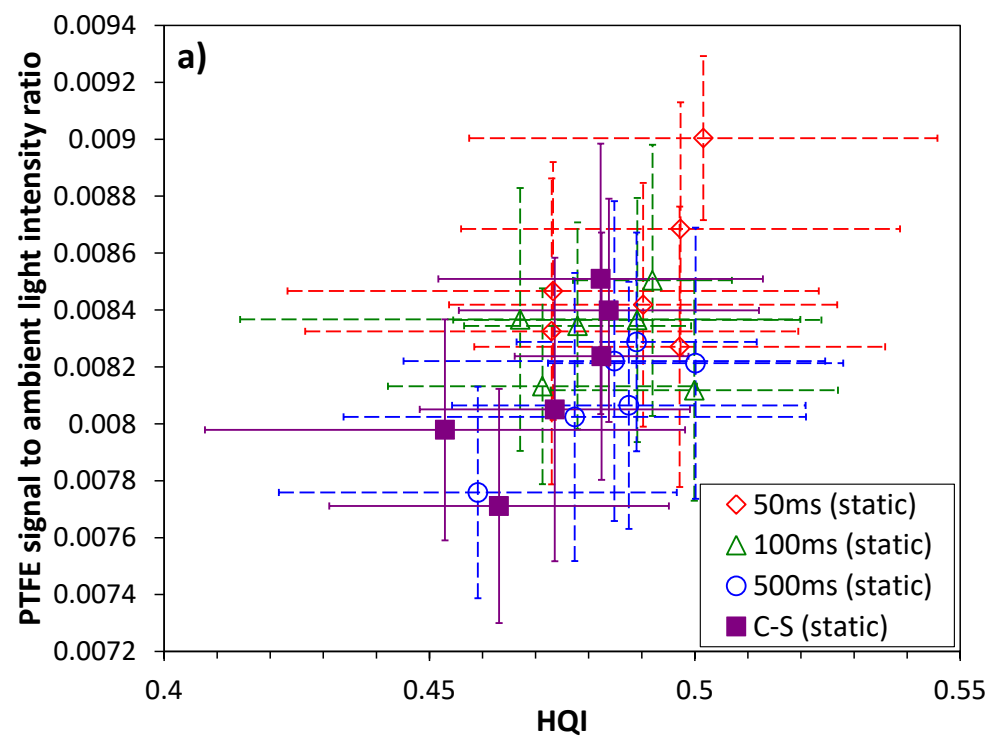




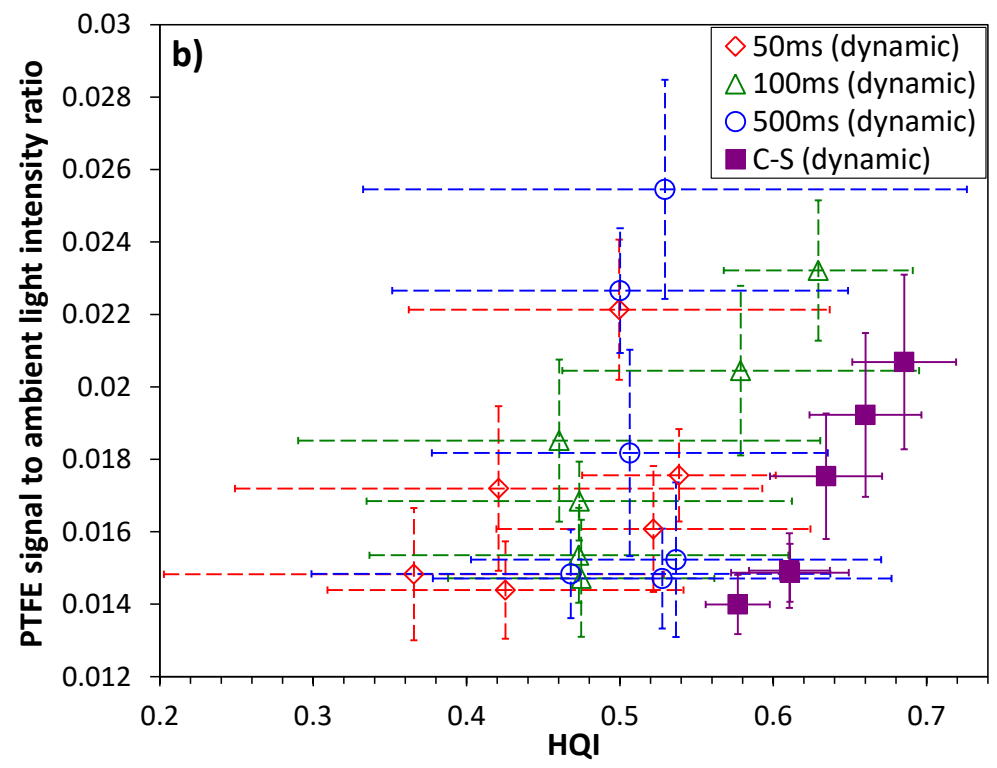

Figure 9: PTFE signal to ambient light intensity ratio plotted against HQI for incandescent light interference under static (a) and dynamic (b) illumination conditions; error bars represent standard deviation of ten individual spectra.

Figure 9a presents the PTFE signal to incandescent light intensity ratio under static illumination conditions. In this configuration all reconstructed spectra are identical within error margins highlighting that the charge-shifting mode performs equally well compared to the conventional read-out at any of the selected sub-acquisition times. Due to the large amount of shot noise being present in the spectra the absolute values for the HQI are generally lower $(\mathrm{HQI}=0.40-0.55)$ compared to the fluorescent light interference $(\mathrm{HQI}=$ 0.94-0.98, see Figure 6a).

Under dynamic illumination conditions the charge-shifting method demonstrates its ability to efficiently reject varying backgrounds resulting in the best spectral reconstruction and lowest variability in this case (see Figure 9b). Independent of the chosen sub-acquisition time the spectral reconstruction performance for the conventional read-out is inferior and shows a larger variation due to residual distortions still being present after spectral reconstruction. Particularly for the charge-shifting spectra it becomes obvious that there is a direct proportionality between achieved HQI and PTFE signal to incandescent light intensity ratio. This effect is expected as better spectral matches can be realized when the contribution of shot noise is reduced.

In contrast to the spectrally narrow distortions introduced by the fluorescent light source (see Figure $5 b$ ) the variations in the reconstructed spectra caused by the incandescent light can be regarded as spectrally broad modulations of the signal background (see Figure 8). For the conventional read-out mode an attempt has therefore been made to remove the spectrally slowly varying baseline by a $7^{\text {th }}$ order polynomial fitting (MATLAB R2013a). Subsequently the spectral reconstruction performance of the corrected spectra was evaluated by means of the HQI. While spectral reconstruction of the distorted spectra 
(recorded in conventional mode under dynamic illumination) show decreased performance and reproducibility compared to the charge-shifting mode (diamond symbols in Figure 10), this difference is eliminated after polynomial baseline correction (triangle symbols in Figure 10). While this approach seems to be successful for a sample with sharp and well-separated Raman bands (as PTFE in this case) it should be noted that in the case of more challenging specimens with complex overlapping Raman bands, e.g. biological tissue or mixtures, there is a risk of adding significant artefacts to the spectra by means of such high-order polynomial fitting and this fix to conventional readout is therefore not amenable to automated data processing as often required, for example, in in-field portable or hand-held Raman instruments, and the charge-shifting mode which does not require this fitting step, would constitute the better performing option.

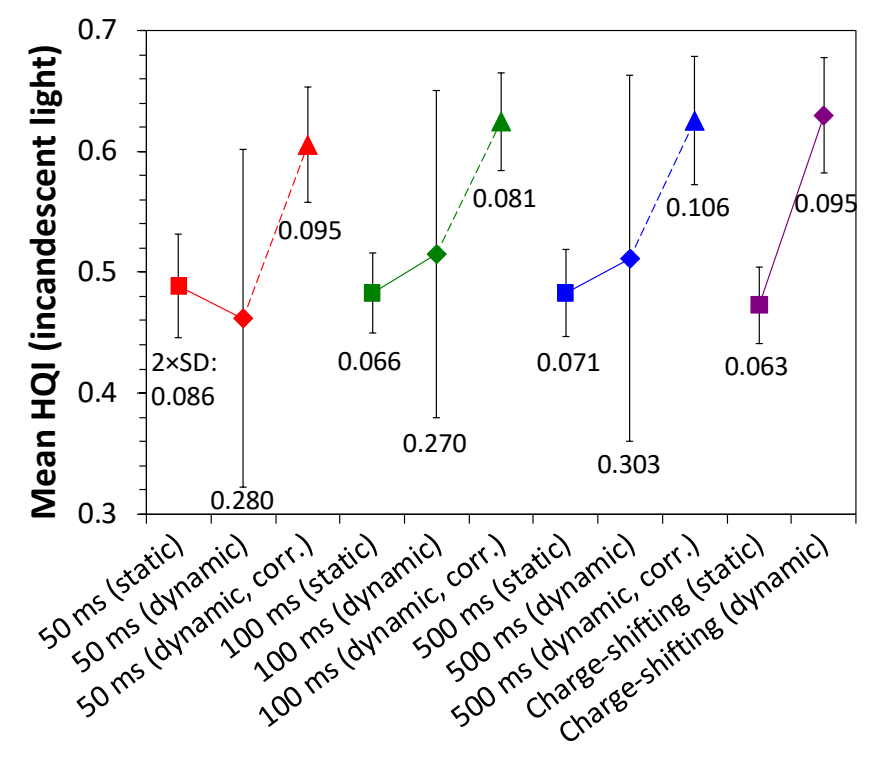

Figure 10: Comparison of mean HQI and standard deviation for 60 spectra recorded under incandescent light illumination in conventional and charge-shifting read-out modes under static illumination (squares), dynamic illumination (diamonds) and dynamic illumination but using 7-th order polynomial background correction (triangles). The numbers given are twice the standard deviation and represent the total length of the error bars.

\subsection{General features of charge-shifting mode}

In both the modes, to address fast variations in ambient light intensities, it is beneficial to use the fastest available CCD modulation frequency. For the conventional read-out in our study this is $7.5 \mathrm{~Hz}$ for $50 \mathrm{~ms}$ sub-acquisition time while in the charge-shifting mode the applied frequency of $1000 \mathrm{~Hz}$ is two orders of magnitude faster and thus providing a clear advantage. The maximum charge-shifting frequency is limited by the time required to shift the number of rows used ( 80 rows taking ca. $680 \mu \mathrm{s}$ ). The 80 pixel shift range also requires about two thirds of the number of horizontal rows to be reserved for charge storage, i.e. 
this area cannot be used for illumination. In future implementations of the technique these issues could be reduced, for example by having an interlaced mask pattern applied directly to the CCD chip covering every second row and then operating a single row shift up and down during the charge-shifting procedure. This would allow using up to $\sim 50 \%$ of the entire CCD area for illumination. This mode of operation is believed would also reduce the distortions currently present due to the spectrograph 'smile effect' potentially making the requirement to record spectra in pairs of " $a$ " and " $b$ " illumination geometries unnecessary.

Tests have been conducted to determine the maximum achievable charge-shifting frequency, which is basically limited by the vertical shifting speed ( $8.25 \mu$ s as fastest possible setting in Andor Solis software) and the actual number of rows to be shifted. After receiving the trigger pulse from the DG645 pulse generator (and an intrinsic electronic delay of $14 \mu \mathrm{s}$ ) the charge is shifted by the specified number of rows. For only one single row shifted up and down the step shift time was determined to be $15 \mu$ s, i.e. the CCD will be ready to receive a light pulse about $29 \mu$ s (delay + shifting time) after the corresponding electronic trigger pulse. If then a light pulse length of $1 \mu$ s was used, the whole cycle (receipt of trigger pulse, delay, single row shift, illumination) would take about $30 \mu \mathrm{s}$. Only after this cycle has been completed the next trigger pulse can follow. Results have demonstrated that in this fully optimized configuration charge-shifting frequencies of up to $32.5 \mathrm{kHz}$ (corresponding to a period between consecutive trigger pulses of $30.8 \mu \mathrm{s}$ ) can be realized with excellent charge confinement to one single row up to the maximum possible number of 65534 cycles (limited by the CCD software). This fast mode of operation does not only open up new avenues in spectroscopic scenarios but also in high-speed imaging applications.

One general limitation of the charge shifting concept is the dynamic range of the CCD and the possibility to saturate the detector when high levels of light strike the CCD. In both the conventional read-out and charge shifting mode the read-out must be performed before saturation occurs and this therefore defines the longest possible acquisition time. Care should therefore be taken for example with highly fluorescent specimens or intense ambient light sources potentially requiring a reduction of the number of charge-shifting cycles or conventional readout sub-acquisition time.

As the removal of ambient light interference is particularly required outside the usual laboratory environment, i.e. where portable instrumentation will be applied, care should also be taken with respect to the amount of data generated as data storage capacity may be limited. The main limitation of the conventional read-out is that fast variations in the spectra can only be captured in kinetic mode but this requires each single sub-acquisition of the kinetic series to be read-out and stored. As access to each individual sub-acquisition is not required in most cases, the charge-shifting technique is beneficial in this respect.

\section{Conclusions}


A charge-shifting CCD read-out approach for the effective rejection of varying ambient light interference from Raman spectra has been implemented and demonstrated using PTFE as a test sample. In comparison with conventional CCD read-out at frequencies of up to $7.5 \mathrm{~Hz}$, the charge-shifting mode (operated at $1000 \mathrm{~Hz}$ ) shows a better ability to deal with irregular fluctuations in the signal background, particularly in the case of ambient light sources with narrow and intense spectral contributions. Library-based spectral matching demonstrates that beside a higher quality of reconstructed spectra the novel approach also benefits from a greater reproducibility of individual spectral reconstructions.

\section{Acknowledgement}

The authors wish to thank the Science and Technology Facilities Council (STFC) for funding this study (Proof-of-Concept project PoCF1516-13). We would like to thank Dr. Bernd Sumpf and Dr. Martin Maiwald (Ferdinand-Braun-Institut, Leibniz-Institut für Höchstfrequenztechnik, Berlin) for the development of the $830 \mathrm{~nm}$ microsystem diode laser and Andor Technology for the provision of the specialized CCD.

\section{References}

(1) L. A. Nafie, J. Raman Spectrosc. 2018, 49, 1874.

(2) E. Widjaja, M. Garland, J. Raman Spectrosc. 2012, 43, 828.

(3) K. L. Gares, K. T. Hufziger, S. V. Bykov, S. A. Asher, J. Raman Spectrosc. 2016, 47, 124.

(4) R. Pilot, J. Raman Spectrosc. 2018, 49, 954.

(5) K. Buckley, J. G. Kerns, J. Vinton, P. D. Gikas, C. Smith, A. W. Parker, P. Matousek, A. E. Goodship, J. Raman Spectrosc. 2015, 46, 610.

(6) K. C. Doty, C. K. Muro, J. Bueno, L. Halámková, I. K. Lednev, J. Raman Spectrosc. 2016, 47, 39.

(7) P. Colomban, J. Raman Spectrosc. 2012, 43, 1529.

(8) J. Wei, A. Wang, J. L. Lambert, D. Wettergreen, N. Cabrol, K. Warren-Rhodes, K. Zacny, J. Raman Spectrosc. 2015, 46, 810.

(9) P. Vandenabeele, H. G. M. Edwards, J. Jehlička, Chem. Soc. Rev. 2014, 43, 2628.

(10) G. Barone, D. Bersani, J. Jehlička, P. P. Lottici, P. Mazzoleni, S. Raneri, P. Vandenabeele, C. Di Giacomo, G. Larinà, J. Raman Spectrosc. 2015, 46, 989.

(11) L. Van de Voorde, M. Vandevijvere, B. Vekemans, J. Van Pevenage, J. Caen, P. Vandenabeele, P. Van Espen, L. Vincze, Spectrochim. Acta B. 2014, 102, 28.

(12) A. Hernanz, J. F. Ruiz-López, J. M. Madariaga, E. Gavrilenko, M. Maguregui, S. Fdez-Ortiz de Vallejuelo, I. Martínez-Arkarazo, R. Alloza-Izquierdo, V. Baldellou-Martínez, R. Viñas-Vallverdú, A. Rubio i Mora, À. Pitarch, A. Giakoumaki, J. Raman Spectrosc. 2014, 45, 1236. 
(13) T. R. Ravindran, A. K. Arora, M. Singh, S. B. Ota, J. Raman Spectrosc. 2012, 44, 108.

(14) R. Scheier, A. Bauer, H. Schmidt, Food Bioprocess Technol. 2014, 7, 2732.

(15) J. C. Carter, S. M. Angel, M. Lawrence-Snyder, J. Scaffidi, R. E.Whipple, J. G. Reynolds, Appl. Spectrosc. 2005, 59, 769.

(16) E. L. Izake, Forensic Sci. Int. 2010, 202, 1.

(17) C. Eliasson, P. Matousek, J. Raman Spectrosc. 2008, 39, 633.

(18) P. Matousek, A. W. Parker, J. Raman Spectrosc. 2007, 38, 563.

(19) J. Desroches, M. Jermyn, K. Mok, C. Lemieux-Leduc, J. Mercier, K. St-Arnaud, K. Urmey, M.-C. Guiot, E. Marple, K. Petrecca, F. Leblond, Biomed. Opt. Express. 2015, 6, 2380.

(20) M. Maiwald, J. Fricke, A. Ginolas, J. Pohl, B. Sumpf, G. Erbert, G. Tränkle, Proc. SPIE. 2014, 8935, $89350 \mathrm{M}-1$.

(21) M. Maiwald, A. Müller, B. Sumpf, G. Tränkle, J. Raman Spectrosc. 2016, 47, 1180.

(22) A. C. De Luca, M. Mazilu, A. Riches, C. S. Herrington, K. Dholakia, Anal. Chem. 2010, 82, 738.

(23) S. Dochow, N. Bergner, C. Matthäus, B. B. Praveen, P. C. Ashok, M. Mazilu, C. Krafft, K. Dholakia, J. Popp, Biomed. Spectrosc. Imaging. 2012, 1, 383.

(24) B. Cletus, W. Olds, P. M. Fredericks, E. Jaatinen, E. L. Izake, J. Forensic Sci. 2013, 58, 1008.

(25) M. Jermyn, J. Desroches, J. Mercier, M.-A. Tremblay, K. St-Arnaud, M.-C. Guiot, K. Petrecca, F. Leblond, J. Biomed. Opt. 2016, 21, 094002-1.

(26) H. P. Povel, H. Aebersold, J. O. Stenflo, Appl. Opt. 1990, 29, 1186.

(27) H. P. Povel, C. U. Keller, I.-A. Yadigaroglu, Appl. Opt. 1994, 33, 4254.

(28) R. Heming, H. Herzog, V. Deckert, DGaO Proceedings. 2008, 109, P33.

(29) J. Mihály, S. Sterkel, H. M. Ortner, L. Kocsis, L. Hajba, É. Furdyga, J. Mink, Croat. Chem. Acta. 2006, 79, 497.

(30) J. D. Rodriguez, B. J. Westenberger, L. F. Buhse, J. F. Kauffman, Anal. Chem. 2011, 83, 4061.

(31) J. Qi, K. L. Bechtel, W.-C. Shih, Biomed. Spectrosc. Imaging. 2014, 3, 359. 
Table 1: Overview of experimental parameters for charge-shifting and conventional CCD read-out modes.

\begin{tabular}{|l|l|l|l|l|}
\hline & \multirow{2}{*}{$\begin{array}{l}\text { Charge- } \\
\text { shifting } \\
\text { mode }\end{array}$} & \multicolumn{3}{|c|}{ Conventional read out } \\
\cline { 3 - 5 } & 1000 & 7.5 & $100 \mathrm{~ms}$ & $500 \mathrm{~ms}$ \\
\hline Frequency (Hz) & 0.319 & 26.2 & 43.1 & 177.2 \\
\hline Laser pulse width (ms) & 0.3 & & 161 \\
\hline Laser pulse delay (ms) & 0.680 & 12 & 29 & 16 \\
\hline No. of accumulations & $2 \times 5000$ & 122 & 74 & 18 \\
\hline Total acquisition time (s) & 10.2 & 16.3 & 13.7 & 10.6 \\
\hline
\end{tabular}

